of legumes and other organisms ${ }^{9}$, and the detailed literature on the various steps in nodulation ${ }^{2}$, highly targeted work such as that of Lodwig et al. will deepen our understanding of how nitrogen-fixing symbioses function. If this is extended to other legumes and other nodulating bacteria, exciting prospects are raised for answering questions ranging from why some legumes cannot nodulate to what distinguishes a pathogen from a symbiont. Above all, perhaps, given their agricultural importance, a better understanding of tropical legumes will assist the management of nitrogen fixation in those areas of the world that need it most.
Janet Sprent is emeritus professor in the School of Life Sciences, University of Dundee,

Dundee DD1 4HN, UK.

e-mail: jisprent@aol.com

1. Lodwig, E. M. et al. Nature 422, 722-726 (2003).

2. Perret, X., Staehelin, C. \& Broughton, W. J. Microbiol. Mol. Biol. Rev. 64, 180-201 (2000).

3. Downie, J. A. \& Parniske, M. Nature 420, 369-370 (2002).

4. Lodwig, E. \& Poole, P. CRC Rev. Plant Sci. 22, 37-78 (2003).

5. Sprent, J. I. Nodulation in Legumes (Royal Botanic Gardens, Kew, 2001).

6. Burdon, J. J., Gibson, A. H., Searle, S. D., Woods, M. J. \& Brockwell, J. J. Appl. Ecol. 36, 398-408 (1999).

7. Roop, R. M. II et al. Vet. Microbiol. 90, 349-363 (2002).

8. Van Elsas, J. D., Turner, S. \& Bailey, M. J. New Phytol. 157, 525-537 (2003).

9. Trevaskis, B. et al. Comp. Funct. Genom. 3, 151-157 (2002).

Earth science

\title{
Roots of the matter
}

\section{B. L. N. Kennett}

\section{How far down does the ancient continental material that constitutes Earth's 'tectosphere' extend? Fresh interpretation of the behaviour of seismic waves helps in reconciling previous estimates.}

0 ver the past three decades there has been vigorous debate over how thick the continents can be - that is, the depth to which the rigid crust and upper mantle reach before meeting convecting mantle that can flow and drive tectonic motion. On page 707 of this issue ${ }^{1}$, Gung and colleagues add new seismological interpretations that go some way to explaining the differing views.

The oldest continental rocks are more than 3.8 billion years old and there are extensive regions of continents, known as 'shields', that are older than 1 billion years. In contrast, the oldest oceanic material is only about 200 million years old, because of the cycle in which oceanic crust is created at mid-ocean ridges and subsequently destroyed as material is returned to depth in subduction zones (particularly around the Pacific). The preservation of old material as the continents move across the Earth's surface due to the relative motions of the tectonic plates is related to what lies beneath: samples brought to the surface through various eruptive processes indicate that there is a significant difference between the continental and oceanic environments.

Based on information from heat flow, geochemistry and the relative delay times of seismic waves in different settings, Jordan ${ }^{2}$ proposed the 'tectosphere' model, in which a zone moves with the motion of the plate lying beneath the old continental shields and would be expected to be about $400 \mathrm{~km}$ thick. More recent assessments of heat-flow data and geochemistry favour a zone no thicker than $250 \mathrm{~km}$. A thickness of $200-250 \mathrm{~km}$ is also consistent with investigations of how the Earth has responded to the removal of the load caused by glaciers, and with regional seismological studies using seismic surface waves. But many seismological models of three-dimensional structure based on global observations would favour a zone extending

Materials science

\section{Mind the graphite gap}

Graphite has diverse applications, ranging from pencils to electronic devices and nuclear reactors. Defects - displacements of carbon atoms - can occur in its structure, which may be beneficial in electronics, but could be dangerous in old-style, air-cooled nuclear reactors such as that pictured. There, the defects store energy and can lead to fire. Quantummechanical computer simulations by Rob Telling and colleagues (Nature Materials doi:10.1038/nmat876; 2003) now show that these defects may be structurally more complex than previously thought.

The structure of graphite itself is essentially simple. It consists of layered sheets known as graphene, each sheet being formed from a planar array of carbon atoms. Defects can form, for example, through the irradiation of graphite in nuclear reactors. This can induce a carbon atom to leave a sheet, forming a 'vacancy defect'. Until now, it had been assumed that the remaining atoms in the sheet are unaffected, and retain a planar configuration.

But the simulations by Telling and colleagues show that the planar state would actually be unstable, and that the atoms surrounding the vacancy are more likely to be displaced out of the plane, very unlike the situation in a flawless sheet. The authors propose that if displaced atoms in two sheets were near to each other, a covalent bond could form between them, effectively bridging the gap.

A vacancy defect can also create another type of flaw, in which the removed carbon atom positions itself between two neighbouring sheets, forming an 'interstitial'. Again, covalent bonds could form. Because the interaction between sheets is usually quite weak — the

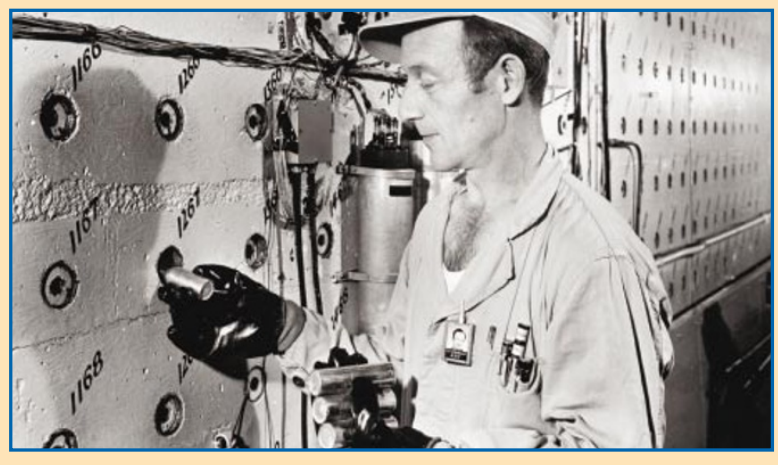

distance between them is $3.35 \AA$, some two-and-a-half times greater than the distance between atoms within a sheet - bonding between them has implications for the properties of the structure.

Although these simulations challenge current ideas about the nature of graphite defects, they are not inconsistent with some of the accepted evidence; they simply provide another explanation for the results. Further research is needed to corroborate Telling and colleagues' theory, but, if validated, the new understanding may help to make the decommissioning of old nuclear reactors safer, and could pave the way to a whole new set of materials based on carbon nanotubes - effectively, rolled-up graphene sheets - which are already provoking great interest and a wealth of research. Jane Morris 
to $400 \mathrm{~km}$, based on the depths at which seismic shear waves travel at elevated speeds - shear waves have their particle motion perpendicular to the direction of propagation. Such higher seismic wave speeds indicate the presence of a region possessing distinct properties, undoubtedly cooler than its surroundings, but also likely to be distinct in composition.

Gung and colleagues ${ }^{1}$ help to reconcile these results. Their work is based on images of Earth structure derived from shear waves with different polarizations. The approach — seismic tomography — is akin to the medical tomography used to study the interior of the human body. In the seismic version, however, illumination of Earth's interior depends on the distribution of earthquakes generating seismic waves and of seismic stations producing high-quality data. Many different classes of wave-propagation phenomena are exploited to improve sampling deep into the Earth. The dominant energy from earthquakes is radiated as shear waves in which the deformation is transverse to their propagation path. Purely horizontally polarized shear waves have somewhat simpler characteristics than those with propagation in a vertical plane and so have been used in many studies. Gung et al. point out that the studies indicating a thick root to the continental shields make extensive use of data from the horizontally polarized waves.

When the authors segregate the information from vertically and horizontally polarized shear waves, they find that the resulting images show significant differences. The structure with fast wave speeds beneath most continental shields extends to at most $250 \mathrm{~km}$ for the vertically polarized waves, whereas the equivalent structures for horizontally polarized waves extend deeper, to nearly $400 \mathrm{~km}$. Gung et al. suggest that this form of seismic anisotropy, with horizontally polarized shear waves travelling faster than those with vertical polarization, is due to the influence of mantle flow in the region between 250 and $400 \mathrm{~km}$. Similar anisotropy has been found under the ocean basins ${ }^{3,4}$ in the depth range from $80 \mathrm{~km}$ to $250 \mathrm{~km}$. The transition to anisotropy caused by mantle flow would then mark the seismic definition of the base of the 'tectosphere', with a depth of no more than $250 \mathrm{~km}$ beneath the ancient continental shields.

In most parts of the world it is difficult to provide any direct test of the results of Gung et al. ${ }^{1}$. But the distribution of earthquakes around the shield of northern Australia, a region that I have studied, is such that higherfrequency seismic waves can be used to probe structure beneath the shield. Here, similar anisotropy is found, with faster travel of horizontally polarized waves ${ }^{5}$ through a zone below $220 \mathrm{~km}$ that produces significant dissipation of wave energy and is hence likely to have lowered viscosity, promoting flow. A complicated structural transition to lowered seismic wave speeds beneath the ancient shield occurs at about $210 \mathrm{~km}$ and has itself been attributed to a different kind of anisotropy ${ }^{6}$. The patterns of seismic anisotropy are subtle and the strong effects associated with polarization are accompanied by variations with the direction of propagation ${ }^{7}$.

Overall, Gung et al. have provided a satisfying way forward in tackling a longstanding puzzle in Earth science. But on the evidence from northern Australia - and as might be expected - the picture will not be straightforward. The seismically defined base of the tectosphere is likely to be quite complex, depending on variations in the local tectonic environment.

B. L. N. Kennett is in the Research School of Earth Sciences, The Australian National University, Canberra, ACT 0200, Australia.

e-mail:brian@rses.anu.edu.au

1. Gung, Y., Romanowicz, B. \& Panning, M. Nature 422, 707-711 (2003).

2. Jordan, T. H. Rev. Geophys. Space Phys. 13, 1-12 (1975).

3. Ekström, G. \& Dziewonski, A. M. Nature 394, 168-172 (1998).

4. Montagner, J.-P. Rev. Geophys. 32, 135-137 (1994).

5. Tong, C., Gudmundsson, O. \& Kennett, B. L. N. J. Geophys. Res. 99, 15783-15797 (1994).

6. Leven, J., Jackson, I. J. \& Ringwood, A. E. Nature 289, 234-236 (1981).

7. Debayle, E. \& Kennett, B. L. N. Earth Planet. Sci. Lett. 184, 339-351 (2000).

\section{Immunology \\ Oxygen and the inflammatory cell}

\section{Carl Nathan}

\section{The discovery that a single protein allows certain immune cells both to respond to low oxygen levels and to induce inflammation may provide a new target for drugs to treat diseases characterized by excessive inflammation.}

n nfected tissues, wounds, rheumatic joints, and parts of tumours that have outgrown their blood supply would seem to have little in common. Yet such sites share two features: they have lower concentrations of oxygen than healthy tissues (they are 'hypoxic'), and they are infiltrated by leukocytes, major cell types of the innate immune system. Writing in Cell, Cramer and colleagues $^{1}$ propose that a single protein mediates both the response of these cells to hypoxia and their ability to participate in inflammation - a coordinated immune response to tissue injuries such as those mentioned above ${ }^{2}$. The protein, hypoxiainducible factor- $1 \alpha$ (HIF- $1 \alpha)$, regulates the expression of at least 30 genes when oxygen levels are $l_{0}{ }^{3}$. Cramer et al. show that HIF- $1 \alpha$ also controls several key aspects of inflammation: the redness and swelling of injured tissues, and the ability of leukocytes to enter these sites. It is striking that a single molecule should emerge as a master regulator in two such diverse and significant settings as hypoxia and inflammation.

Leukocytes come in various guises, two major subtypes being neutrophils and macrophages. In one of the earliest responses to injury, neutrophils in an affected tissue's venules - small blood vessels that receive input from capillaries - stop flowing with the blood. Instead, they stick to the inside of the venules and form clumps that can grow to occlude the vessel, reducing blood flow. In addition, some neutrophils cross the vessel wall, migrating into the tissue. The reduced blood flow and increased cell numbers lead to local depletion of oxygen. Then, macrophage precursor cells arrive.

Hypoxia causes macrophages, neutro- phils and other cells to activate a variety of their genes, including that encoding vascular endothelial growth factor (VEGF), which makes blood vessels leaky ${ }^{4}$. This gene activation is achieved by means of HIF- $1 \alpha$, which binds to the promoters (regulatory elements) of target genes. To do so, HIF- $1 \alpha$ must first bind its partner, HIF-1 $\beta$, and this interaction is controlled by oxygen levels. When oxygen is abundant, there is little HIF- $1 \alpha-$ it is destroyed under the direction of the von Hippel-Lindau (VHL) protein - and what there is can't bind HIF-1 $\beta$. At low oxygen levels these twin restraints are lifted ${ }^{5}$ (Fig. 1, overleaf).

Given that HIF- $1 \alpha$ is active in hypoxic leukocytes, Cramer et al. ${ }^{1}$ wanted to find out whether it is needed in inflammation. To study the function of a protein, researchers often inactivate its gene in experimental organisms such as mice, to see what happens. But disrupting the HIF- $1 \alpha$ gene kills mice when they are embryos, precluding any subsequent study of inflammation. So Cramer et al. used a special technique in mice to delete the protein only in leukocytes. The approach involved using the promoter of the lysozyme gene, which is largely active only in leukocytes, to switch on an enzyme that inactivates the HIF-1 $\alpha$ gene.

The researchers found that isolated macrophages and neutrophils from the mutant mice had reduced levels of VEGF and phosphoglycerate kinase compared with wild-type cells. The latter enzyme is needed to generate ATP, the main cellular energy store, from glucose by glycolysis; this oxygen-independent process is the predominant means by which leukocytes generate energy. As might be expected, 